\title{
Characterization of Patients with Ankylosing Spondylitis Receiving Secukinumab and Reasons for Initiating Treatment: A US Physician Survey and Retrospective Medical Chart Review
}

\author{
Reeti Joshi ${ }^{1} \cdot$ Dominick Latremouille-Viau $^{2} \cdot$ Mark K. Meiselbach $^{3} \cdot$ Jipan Xie $^{4} \cdot$ Yujin Park $^{5} \cdot$ Prashanth Sunkureddi $^{6}$
}

Published online: 7 January 2019

(c) The Author(s) 2019

\begin{abstract}
Objectives To characterize US patients with ankylosing spondylitis (AS) who were treated with secukinumab and to assess rheumatologist-reported reasons for prescribing treatment in clinical practice.

Methods This descriptive analysis of data from a US retrospective medical chart review included patients aged $\geq 18$ years diagnosed with AS who initiated secukinumab after 15 January 2016. Eligible rheumatologists used online forms to collect patient demographics, disease characteristics, co-morbidity profile, and treatment history prior to or on the index date, defined as the date of the first secukinumab prescription recorded in the medical chart. Information on physician-level characteristics and reasons for secukinumab prescription and dosing were also collected.

Results Medical charts from 78 patients with AS who initiated secukinumab were reviewed by 25 rheumatologists between 7 July 2017 and 11 August 2017. Overall, 76.9\% of patients were male, mean (SD) age was 39.8 (10.8) years, and 34.6\% were biologic naïve. The most common reasons for secukinumab initiation among biologic-naïve and biologic-experienced patients, respectively, were efficacy/effectiveness $(77.8 \%)$ and failure of other prior biologics (84.3\%). Nearly all patients (94.9\%) received a loading dose, including $150 \mathrm{mg}$ every week (39.7\%), $300 \mathrm{mg}$ every week (53.8\%), and other (1.3\%). Overall, 73 patients $(93.6 \%)$ received $\geq 1$ maintenance secukinumab dose, of whom $56.2 \%$ and $43.8 \%$ received $150 \mathrm{mg}$ and $300 \mathrm{mg}$, respectively, every 4 weeks.

Conclusions In this US medical chart review of patients with AS who initiated secukinumab, approximately one-third were biologic naïve, and secukinumab efficacy/effectiveness and failure of other biologics were the most common reasons for initiating secukinumab.
\end{abstract}

Electronic supplementary material The online version of this article (https://doi.org/10.1007/s40801-018-0146-9) contains supplementary material, which is available to authorized users.

Reeti Joshi

Baylor College of Medicine, Houston, TX, USA

2 Analysis Group, Inc., Montreal, QC, Canada

3 Analysis Group, Inc., Boston, MA, USA

4 Analysis Group, Inc., Los Angeles, CA, USA

5 Novartis Pharmaceuticals Corporation, East Hanover, NJ, USA

6 University of Texas Medical Branch, Galveston, TX, USA

\section{Key Points}

In this medical chart review of US patients with ankylosing spondylitis, approximately one-third of patients (34.6\%) were biologic naïve, and another $53.8 \%$ of patients had received only one biologic prior to initiating secukinumab, suggesting that rheumatologists are prescribing secukinumab early in the treatment algorithm when considering biologic therapy.

The most common reasons for secukinumab initiation among biologic-naïve and biologic-experienced patients, respectively, were efficacy/effectiveness of secukinumab (77.8\%) and failure of other prior biologics (84.3\%).

These findings will provide information to rheumatologists on how secukinumab is used by their peers for the treatment of ankylosing spondylitis in US clinical practice. 


\section{Introduction}

Ankylosing spondylitis (AS) is a chronic, immune-mediated disease characterized by involvement of the axial skeleton and sacroiliac joints $[1,2]$. Inflammation of the vertebrae is often associated with chronic pain and stiffness of the entire spine and sacroiliac joints, and in severe cases can lead to structural changes in the spine-including fusion of the spine and sacroiliac joints-and new bone formation [1, 2]. AS has an estimated prevalence of $0.2-0.5 \%$ in the US and is associated with considerable physical, emotional, and economic burden [3-5].

The goals of treatment in patients with AS are to reduce and/or control inflammation and symptoms of pain, stiffness, and fatigue; maintain spinal flexibility and normal posture; reduce functional limitations; preserve or normalize social participation; maintain work ability; and decrease complications of disease (e.g., uveitis) [6, 7]. Current treatment guidelines recommend nonsteroidal anti-inflammatory drugs (NSAIDs) as first-line therapy in patients with active AS who have pain and stiffness. In addition, local glucocorticoids may be used for patients with peripheral arthritis, enthesitis, or isolated sacroiliitis. Analgesics (e.g., acetaminophen and opioids) may be considered in patients in whom previously recommended treatments have failed and resulted in residual pain, are contraindicated, and/or are poorly tolerated. In patients with active AS despite NSAID treatment, use of a biologic is recommended. Current practice suggests use of a tumor necrosis factor inhibitor (TNFi) as first-line biologic therapy; however, approximately $40 \%$ of patients do not achieve a response, as defined by the Assessment of SpondyloArthritis international Society criteria for $20 \%$ improvement (ASAS 20) [8]. If the initial TNFi therapy fails, switching to another TNFi or an interleukin (IL)-17 inhibitor should be considered [7]. Although switching TNFis may be effective for some patients, subsequent TNFis may not be as effective [9-11], highlighting the unmet patient needs for biologics with different mechanisms of action.

Secukinumab is a fully human anti-IL-17A monoclonal antibody and is the first and only non-TNFi approved for the treatment of AS in the US. With patients and physicians having more options available for treatment, it is important to understand why therapies are being started or stopped and how they are being prescribed; however, there are a limited number of studies describing why or how secukinumab is being prescribed in real-world settings. The objectives of this analysis were to describe the clinical and treatment characteristics of patients with AS who were prescribed secukinumab in US clinical practice and to describe rheumatologist-reported reasons for prescribing secukinumab.

\section{Materials and Methods}

\subsection{Data Source and Study Design}

This was a retrospective medical chart review of patients in the US diagnosed with AS and aged $\geq 18$ years who initiated secukinumab treatment after its approval date of 15 January 2016. Rheumatologists who were from different geographic areas and represented different demographics and practice settings were recruited from an external vendor to participate in this study. The selected vendor has the largest panel of medical specialists in the US. Physicians in this panel cover all states of the US and have similar age, gender, and practice type distribution to the American Medical Association (AMA)-registered physicians. The vendor's access to panelists is facilitated by the online services provided by the vendor to physicians, such as medical education, ethical drug promotion, clinical development, job recruitment, and clinic appointment services. Rheumatologists were screened based on the following eligibility criteria: (1) completed their residency and fellowship training; (2) treated $\geq 1$ patient with AS in the 12 months prior to the chart review; and (3) had prescribed secukinumab for the treatment of AS. Eligible rheumatologists were then invited to provide patient information using an online chart abstraction form.

This study was designed and implemented in accordance with the Guidelines for Good Pharmacoepidemiology Practices of the International Society for Pharmacoepidemiology, the Strengthening the Reporting of Observational Studies in Epidemiology (STROBE) guidelines, and with the ethical principles laid down in the Declaration of Helsinki [12, 13]. An institutional review board exemption was obtained for the study prior to initiation of data collection.

\subsection{Study Population}

Eligible patients were required to meet the following criteria: (1) received a diagnosis of AS; (2) were prescribed secukinumab for the treatment of AS by a participating rheumatologist in a real-world setting (i.e., outside of an interventional clinical trial) on or after 15 January 2016; (3) were aged $\geq 18$ years at the time of secukinumab initiation; and (4) had medical records containing information on AS diagnosis, co-morbidities, and treatment history prior to the secukinumab initiation that were accessible to the participating rheumatologists.

\subsection{Measures and Outcomes}

The chart abstraction form was used to collect patient-level information abstracted by rheumatologists from eligible patients' medical charts, such as demographics (age, sex, 
race, and insurance type), disease characteristics (disease duration, physician-assessed disease severity, AS symptoms experienced, and co-morbidities), treatment history (previous treatments and rheumatologist-reported reasons for discontinuation of treatment immediately preceding secukinumab), and characteristics of secukinumab use (duration of treatment, dosing, current secukinumab treatment status, rheumatologist-reported reasons for secukinumab prescription, and reasons for secukinumab discontinuation [if applicable]). The screening section was used both to confirm that participating rheumatologists had $\geq 1$ chart for a patient with AS that met all study selection criteria and to collect physician-level data such as demographics (age and sex), practice characteristics (type of practice and region of practice), and experience treating patients with AS (years of experience, number of patients with AS seen in the previous year, number of AS charts contributed to the study, and tools and references used to assist AS diagnosis).

\subsection{Data Analysis}

Descriptive statistics are shown for all patient demographics, disease characteristics, treatment history, and characteristics of secukinumab use, as well as physician-level characteristics. Rheumatologist-reported reasons for secukinumab prescription were also examined separately among biologicnaïve and biologic-experienced patients. Categorical variables were summarized using frequency counts and percentages. Continuous variables were summarized by means and SDs or medians and interquartile ranges (IQRs).

All data analyses were conducted using Statistical Analysis System 9.4 software.

\section{Results}

\subsection{Rheumatologist Characteristics}

Medical charts for 78 patients with AS who initiated secukinumab were reviewed by 25 rheumatologists between 7 July 2017, and 11 August 2017. On average, rheumatologists contributed a mean (SD) of 3.1 (1.6) AS medical charts to the analysis.

Characteristics of the participating rheumatologists are presented in Table 1. Overall, the rheumatologists had been in practice for a mean (SD) of 12.8 (9.2) years; 19 of 25 rheumatologists were from a private practice setting and 11 of 25 were from the Northeast region of the US. Most rheumatologists $(n=19)$ used established criteria to assist in the diagnosis of AS, including the ASAS criteria $(n=15)$, the 1984 modified New York criteria for AS $(n=13)$, or both $(n=9)$. In addition, nearly all rheumatologists $(n=24)$ referenced published international treatment guidelines to assist with AS treatment, including those published by the American College of Rheumatology (ACR) ( $n=22)$, ASAS/European League Against Rheumatism (EULAR) $(n=9)$, or both $(n=7)$.

\subsection{Patient Demographic and Disease Characteristics at the Time of Secukinumab Initiation}

Among the 78 patients with AS whose charts were reviewed, $76.9 \%$ were male and the mean (SD) age was 39.8 (10.8) years (Table 2). At the time of secukinumab initiation, $14.1 \%$ of patients had a disease duration of $<1$ year and $7.7 \%$ had a duration of $>10$ years.

Table 1 Characteristics of rheumatologists participating in the medical chart review of patients with AS $(N=25)$

\begin{tabular}{|c|c|}
\hline Characteristic & $\begin{array}{l}\text { Rheuma- } \\
\text { tologists } \\
(N=25)\end{array}$ \\
\hline Age, mean (SD), years & $45.8(12.4)$ \\
\hline Male, $n$ & 18 \\
\hline \multicolumn{2}{|l|}{ Type of practice, $n$} \\
\hline Private practice & 19 \\
\hline Academic institution & 6 \\
\hline \multicolumn{2}{|l|}{ Region of practice, $n$} \\
\hline Northeast & 11 \\
\hline Midwest & 4 \\
\hline South & 6 \\
\hline West & 4 \\
\hline Years in practice, mean $(\mathrm{SD})$ & $12.8(9.2)$ \\
\hline $\begin{array}{l}\text { No. of AS patient medical charts contributed by physi- } \\
\text { cian, mean (SD) }\end{array}$ & $3.1(1.6)$ \\
\hline \multicolumn{2}{|l|}{ Tools referenced to assist AS diagnosis, $n^{\mathrm{a}}$} \\
\hline New York criteria & 13 \\
\hline ASAS criteria & 15 \\
\hline New York criteria and ASAS criteria & 9 \\
\hline Other & 0 \\
\hline No criteria & 6 \\
\hline \multicolumn{2}{|l|}{ AS treatment guidelines referenced, $n^{\mathrm{b}}$} \\
\hline $\mathrm{ACR}$ & 22 \\
\hline ASAS/EULAR & 9 \\
\hline ACR and ASAS/EULAR & 7 \\
\hline Other & 0 \\
\hline No guidelines & 1 \\
\hline
\end{tabular}

ACR American College of Rheumatology, AS ankylosing spondylosis, ASAS Assessment of SpondyloArthritis international Society, EULAR European League Against Rheumatism

${ }^{a}$ Multiple tools could be selected by a single physician except when "no criteria" was selected

${ }^{\mathrm{b}}$ Multiple guidelines could be selected by a single physician except when "no guidelines" was selected 
Overall, most patients $(91.0 \%)$ had moderate or severe rheumatologist-assessed AS disease severity. The most common symptoms were lower back pain $(91.0 \%)$, morning stiffness (83.3\%), and limited spinal mobility/range of motion (61.5\%). Nearly two-thirds of patients (65.4\%) had co-morbidities, the most common of which were hypertension $(25.6 \%)$, anemia (20.5\%), digestive disorders $(15.4 \%)$, and anxiety $(11.5 \%) ; 5.1 \%$ of patients also had psoriasis (Table 2).

\subsection{AS Treatment History}

All 78 patients with AS received treatment prior to initiating secukinumab (Table 3 ). The median (IQR) time from initiation of patients' first treatment to initiation of secukinumab was 18.1 (9.0-33.1) months, and patients received a mean (SD) of 2.5 (1.3) treatments prior to initiating secukinumab. Patients received a mean (SD) of $0.8(0.7)$ prior biologics at any time prior to secukinumab initiation, including 42 patients $(53.8 \%)$ who received one prior biologic, eight $(10.3 \%)$ who received two prior biologics, and one $(1.3 \%)$ who received $\geq$ three prior biologics. Over one-third of patients $(n=27 ; 34.6 \%)$ were biologic naïve. Etanercept (29.5\%) and adalimumab (28.2\%) were the most common biologics used prior to secukinumab. NSAIDs $(52.6 \%)$ and biologics $(51.3 \%)$ were the most commonly used classes of treatments directly preceding secukinumab. The most common rheumatologist-reported reasons for discontinuation of treatment directly preceding secukinumab were lack/loss of efficacy/effectiveness $(66.7 \%)$, disease progression $(30.8 \%)$, and availability of new treatment (23.1\%) (Fig. 1a).

\subsection{Rheumatologist-Reported Reasons for Prescription of Secukinumab and Characteristics of Secukinumab Use}

The most common rheumatologist-reported reasons for secukinumab prescription were efficacy/effectiveness of secukinumab $(70.5 \%)$, failure of other prior biologics (55.1\%), and secukinumab's status as a newly available agent with a new mechanism of action (52.6\%) (Fig. 1b). When stratified by use of prior biologics (i.e., whether biologic naïve or biologic experienced), the most common reason for secukinumab prescription in biologic-naïve patients was efficacy/effectiveness of secukinumab $(77.8 \%)$, followed by favorable safety profile of secukinumab $(51.9 \%)$, and perceived sustained/long-lasting efficacy of secukinumab (51.9\%) (Supplemental Fig. S1). Failure of other prior biologics $(84.3 \%)$, efficacy/effectiveness of secukinumab (66.7\%), and secukinumab's status as a newly available agent with a new mechanism of action (62.7\%) were
Table 2 Demographic and disease characteristics at the time of secukinumab initiation of patients with AS $(N=78)$

\begin{tabular}{|c|c|}
\hline Characteristic & Patients with AS $(N=78)$ \\
\hline \multicolumn{2}{|l|}{ Age } \\
\hline Mean (SD), years & $39.8(10.8)$ \\
\hline Median (IQR), years & $38.0(32.0-45.0)$ \\
\hline Male, $n(\%)$ & $60(76.9)$ \\
\hline \multicolumn{2}{|l|}{ Race/ethnicity, $n(\%)$} \\
\hline White/non-Hispanic & $54(69.2)$ \\
\hline Hispanic & $13(16.7)$ \\
\hline Black/non-Hispanic & $8(10.3)$ \\
\hline Asian/Pacific Islander & $3(3.8)$ \\
\hline \multicolumn{2}{|l|}{ Insurance type, $n(\%)$} \\
\hline Commercial/private & $56(71.8)$ \\
\hline Medicaid & $13(16.7)$ \\
\hline Medicare & $9(11.5)$ \\
\hline Military & $1(1.3)$ \\
\hline No insurance & $1(1.3)$ \\
\hline Unknown/not sure & $1(1.3)$ \\
\hline \multicolumn{2}{|l|}{ Duration of AS, $n(\%)$} \\
\hline$<1$ year & $11(14.1)$ \\
\hline $1-2$ years & $26(33.3)$ \\
\hline $3-5$ years & $22(28.2)$ \\
\hline $6-10$ years & $13(16.7)$ \\
\hline$>10$ years & $6(7.7)$ \\
\hline \multicolumn{2}{|l|}{ Physician-assessed disease severity, $n(\%)$} \\
\hline Mild & $7(9.0)$ \\
\hline Moderate & $56(71.8)$ \\
\hline Severe & $15(19.2)$ \\
\hline AS symptoms experienced, $n(\%)^{\mathrm{a}}$ & $78(100)$ \\
\hline Lower back pain & $71(91.0)$ \\
\hline Morning stiffness & $65(83.3)$ \\
\hline $\begin{array}{l}\text { Limited spinal mobility and range of } \\
\text { motion }\end{array}$ & $48(61.5)$ \\
\hline Fatigue (e.g., low energy/tired) & $47(60.3)$ \\
\hline Nocturnal pain & $45(57.7)$ \\
\hline Neck pain & $38(48.7)$ \\
\hline Hip pain & $26(33.3)$ \\
\hline Enthesitis & $23(29.5)$ \\
\hline Peripheral arthritis & $20(25.6)$ \\
\hline Dactylitis & $10(12.8)$ \\
\hline Inflammatory eye disease & $6(7.7)$ \\
\hline Other $^{\mathrm{b}}$ & $1(1.3)$ \\
\hline \multicolumn{2}{|l|}{ Co-morbidities, $n(\%)^{\mathrm{c}}$} \\
\hline Hypertension & $20(25.6)$ \\
\hline Anemia & $16(20.5)$ \\
\hline Digestive disorders & $12(15.4)$ \\
\hline Anxiety & $9(11.5)$ \\
\hline Hyperlipidemia & $8(10.3)$ \\
\hline Depression & $6(7.7)$ \\
\hline Inflammatory eye diseases (e.g., uveitis) & $5(6.4)$ \\
\hline Crohn disease & $4(5.1)$ \\
\hline
\end{tabular}


Table 2 (continued)

\begin{tabular}{lc}
\hline Characteristic & Patients with AS $(N=78)$ \\
\hline Diabetes & $4(5.1)$ \\
Psoriasis & $4(5.1)$ \\
Ulcerative colitis $^{\text {Malignancy }}$ & $4(5.1)$ \\
M $^{\text {M }}$ & $2(2.6)$ \\
\hline
\end{tabular}

$A S$ ankylosing spondylosis

aultiple AS symptoms could be selected for each patient except when "None of the above" was selected

b Other symptoms included "psoriasis"

${ }^{\mathrm{c}}$ Multiple co-morbidities could be selected for each patient except if "none of the above" was selected

"Malignancy included "melanoma"

the most common reasons for secukinumab prescription in biologic-experienced patients.

At the time of chart abstraction, patients had received secukinumab for a mean (SD) of 4.7 (4.6) months (Table 4). Only $39.7 \%$ of patients with AS received an initial loading dose of $150 \mathrm{mg}$ every week, while $53.8 \%$ received an initial loading dose of $300 \mathrm{mg}$ every week and $5.1 \%$ did not receive a loading dose. Of the 73 patients who received an initial maintenance dose of secukinumab, more than half
(56.2\%) received an initial maintenance dose of $150 \mathrm{mg}$ every 4 weeks, with the remaining $43.8 \%$ of patients receiving an initial maintenance dose of $300 \mathrm{mg}$ every 4 weeks. Nearly all patients $(94.9 \%)$ were still receiving secukinumab at the time of chart abstraction, while three patients (3.8\%) had discontinued treatment, and one patient (1.3\%) had an unknown treatment status. Among the three patients who discontinued treatment, one patient discontinued due to lack of efficacy/effectiveness, one due to patient request and lack of efficacy/effectiveness, and one due to death unrelated to secukinumab treatment.

\section{Discussion}

In this retrospective chart review of 78 patients who initiated secukinumab for the treatment of AS in US clinical practice, nearly all patients $(91.0 \%)$ had moderate or severe disease as assessed by their rheumatologist, approximately threequarters had received an AS diagnosis $\leq 5$ years ago, and hypertension and anemia were the most commonly reported co-morbidities. Prior to secukinumab initiation, NSAIDs and biologics were the most common classes of medication used in these patients; approximately one-third of patients were biologic naïve. There are a limited number of real-world
Table 3 Treatment history of patients with AS $(N=78)$

\begin{tabular}{lc}
\hline Characteristic & Patients with AS $(N=78)$ \\
\hline Received treatment prior to secukinumab, $n(\%)$ & $78(100.0)$ \\
Time since initiation of first treatment to initiation of secukinumab, median & $18.1(9.0-33.1)$ \\
$\quad$ (IQR), months & \\
No. of treatments received prior to secukinumab ${ }^{\mathrm{a}}$ & \\
Mean (SD) & $2.5(1.3)$ \\
$1, n(\%)$ & $16(20.5)$ \\
$2, n(\%)$ & $32(41.0)$ \\
$\geq 3, n(\%)$ & $30(38.5)$ \\
No. of biologics received prior to secukinumab ${ }^{\mathrm{a}}$ & \\
Mean (SD) & $0.8(0.7)$ \\
$1, n(\%)$ & $42(53.8)$ \\
$2, n(\%)$ & $8(10.3)$ \\
$\geq 3, n(\%)$ & $1(1.3)$ \\
Treatments directly preceding secukinumab, $n(\%)^{\mathrm{b}}$ & \\
NSAIDs & $41(52.6)$ \\
Any biologic & $40(51.3)$ \\
Nonbiologic DMARDs & $22(28.2)$ \\
Analgesics and opioids & $6(7.7)$ \\
Glucocorticoids & $5(6.4)$ \\
\hline
\end{tabular}

$A S$ ankylosing spondylitis, DMARD disease-modifying antirheumatic drug, IQR interquartile range, NSAID nonsteroidal anti-inflammatory drug

${ }^{a}$ The number of treatments received prior to secukinumab was calculated among patients who had received $\geq 1$ treatment prior to secukinumab. The number of all previous distinct treatments was considered, regardless of treatment combinations

${ }^{\mathrm{b}}$ Multiple treatments directly preceding secukinumab could be selected for each patient 
Fig. 1 a Rheumatologistreported reasons for discontinuing the treatment that directly preceded secukinumab initiation in patients with AS and $\mathbf{b}$ rheumatologist-reported reasons for secukinumab prescription in patients with AS $(N=78)$. $A S$ ankylosing spondylitis
A

Rheumatologist-Reported Reasons for Discontinuation of Treatment Directly Preceding Secukinumab Initiation

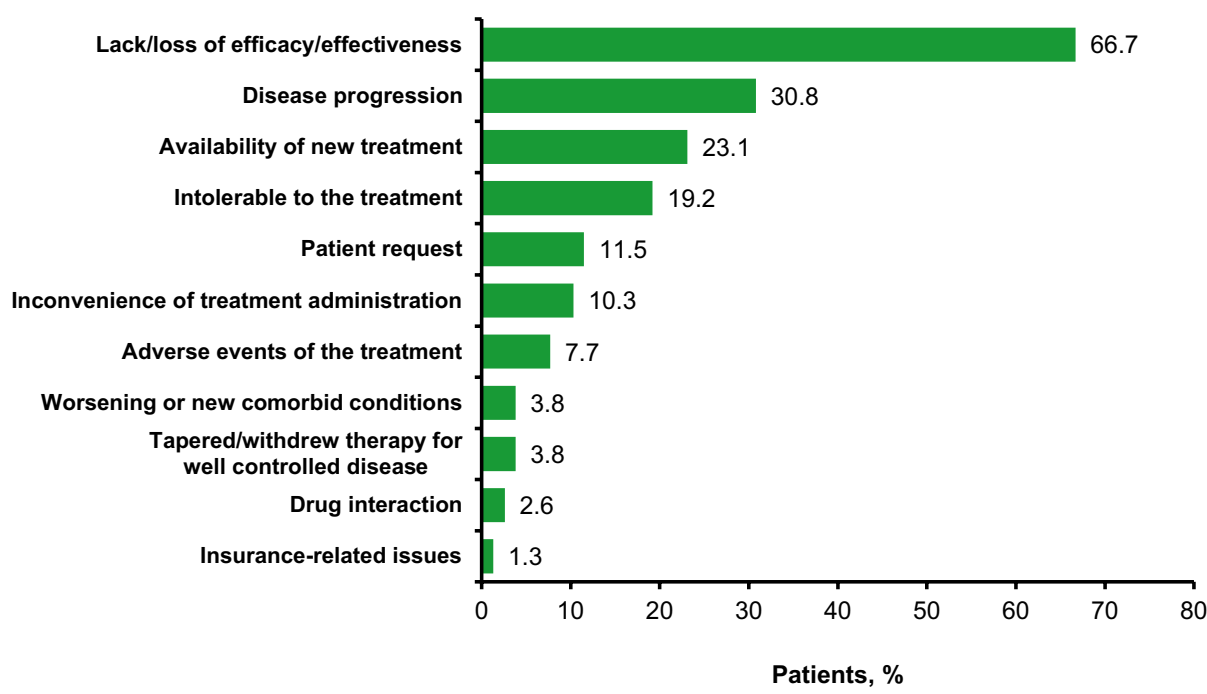

B

B Rheumatologist-Reported Reasons

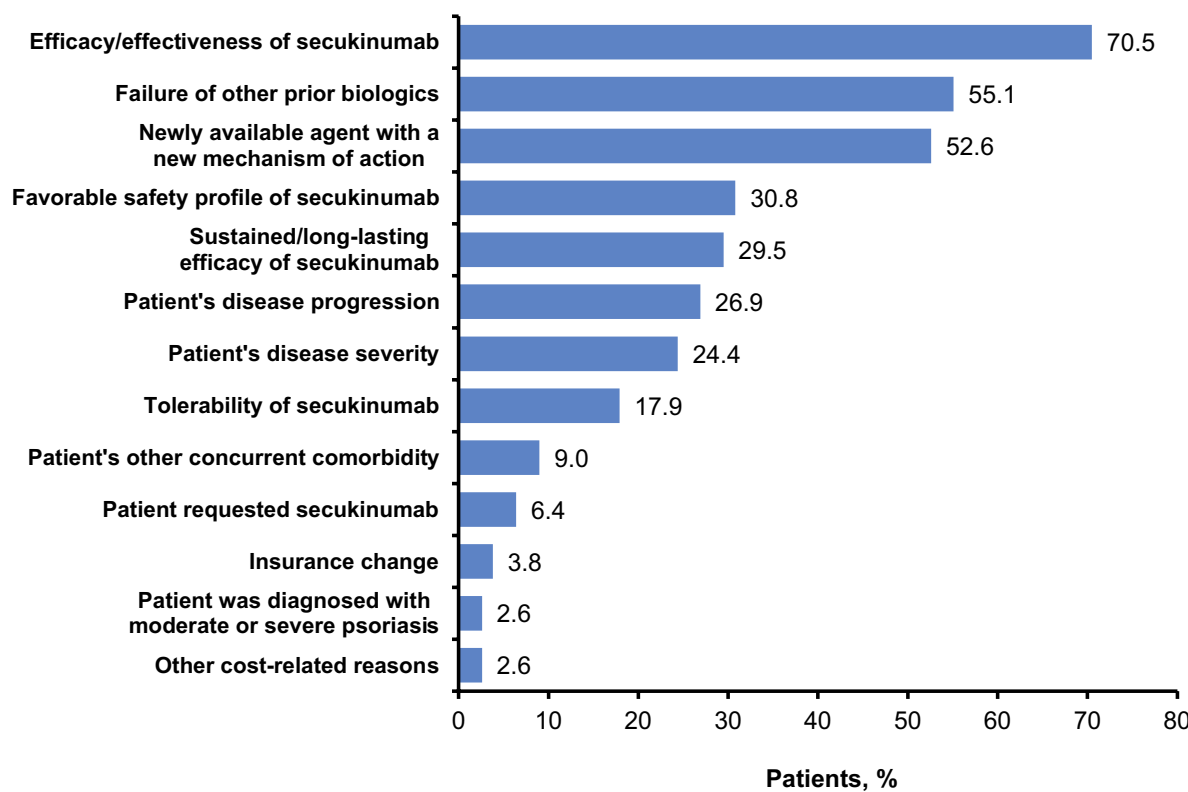

studies that have characterized patients in clinical practice who initiated secukinumab for AS. A cross-sectional, webbased panel survey evaluated real-world treatment experience and satisfaction with secukinumab in 200 US patients with AS, $98.5 \%$ of whom had received some treatment for AS prior to initiation of secukinumab, and included patients who were primarily male $(60.0 \%)$ and White $(66.0 \%)$, with a mean (SD) age of 34.4 (10.6) years [14]. A retrospective analysis from the Symphony Health Solutions, LLC, national medical claims and pharmacy database described the demographic and clinical characteristics of 543 patients with AS who initiated secukinumab in routine clinical practice between 15 January 2016, and 31 December 2016 [15]. Compared with the Symphony database, which also did not collect information on HLA-B27 and based diagnoses on ICD-9-CM or ICD-10 codes, the patients in our chart review were younger (39.8 vs. 46.4 years) and more likely to be male ( $76.9 \%$ vs. $48.4 \%$ ). However, both cohorts had similar proportions of patients with hypertension as the top co-morbidity $(\approx 25 \%$ ), as well as the proportion of patients who were biologic naïve prior to initiation of secukinumab ( $\approx 34 \%$ ). Due to the limitations of healthcare claims data, no information on disease severity was captured in the Symphony database; thus, no comparisons could be made 
Table 4 Characteristics of secukinumab use of patients with AS $(N=78)$

\begin{tabular}{|c|c|}
\hline Characteristic & $\begin{array}{l}\text { Patients with } \\
\text { AS }(N=78)\end{array}$ \\
\hline \multicolumn{2}{|l|}{ Duration of treatment with secukinumab ${ }^{\mathrm{a}}$} \\
\hline Mean (SD), months & $4.7(4.6)$ \\
\hline Median (IQR), months & $3.5(1.0-6.0)$ \\
\hline \multicolumn{2}{|l|}{ Dose } \\
\hline \multicolumn{2}{|l|}{ Initial loading dose, $n(\%)$} \\
\hline 150 mg every week ${ }^{b}$ & $31(39.7)$ \\
\hline $300 \mathrm{mg}$ every week ${ }^{\mathrm{c}}$ & $42(53.8)$ \\
\hline Other $^{\mathrm{d}}$ & $1(1.3)$ \\
\hline No loading dose & $4(5.1)$ \\
\hline \multicolumn{2}{|l|}{ Received maintenance dose, $n(\%)$} \\
\hline \multicolumn{2}{|l|}{ Initial maintenance dose $\mathrm{e}^{\mathrm{e}}$} \\
\hline 150 mg every 4 weeks & $41(56.2)$ \\
\hline 300 mg every 4 weeks & $32(43.8)$ \\
\hline \multicolumn{2}{|l|}{ Current use of secukinumab, $n(\%)$} \\
\hline Patient still receiving secukinumab & $74(94.9)$ \\
\hline Patient discontinued secukinumab & $3(3.8)$ \\
\hline \multicolumn{2}{|c|}{ Reason for secukinumab discontinuation, $n(\%)^{\mathrm{f}}$} \\
\hline Lack/loss of efficacy/effectiveness & $2(66.7)$ \\
\hline Patient request & $1(33.3)$ \\
\hline Other $^{\mathrm{g}}$ & $1(33.3)$ \\
\hline Unknown status of secukinumab use & $1(1.3)$ \\
\hline
\end{tabular}

$A S$ ankylosing spondylitis, IQR interquartile range

${ }^{a}$ Duration of treatment with secukinumab was calculated among all patients; the calculated duration underestimates the true duration because most patients were still receiving secukinumab at the time of the survey

${ }^{\mathrm{b}}$ Among the 31 patients who initiated secukinumab at $150 \mathrm{mg}$ every week, 24 received an initial maintenance dose of $150 \mathrm{mg}$ every 4 weeks, five received $300 \mathrm{mg}$ every 4 weeks, and two did not remain on the therapy long enough to receive maintenance treatment

${ }^{c}$ Among the 42 patients who initiated secukinumab at $300 \mathrm{mg}$ every week, 14 received an initial maintenance dose of $150 \mathrm{mg}$ every 4 weeks, 26 received $300 \mathrm{mg}$ every 4 weeks, and two did not remain on the therapy long enough to receive maintenance treatment

dOther initial loading dose included "methotrexate"

${ }^{\mathrm{e}}$ The proportions of initial maintenance doses were calculated among patients who received a maintenance dose

${ }^{\mathrm{f}}$ The proportions of reasons for discontinuation were calculated among patients who discontinued secukinumab. Multiple reasons could be selected for each patient

gOther reason included "patient deceased"

across real-world cohorts regarding clinical characteristics. Although in our study disease severity was assessed on the basis of the participating rheumatologists' clinical expertise, the high proportion of patients with moderate and severe disease is consistent with patients treated with secukinumab enrolled in phase III randomized controlled trials [16-18]. In contrast with patients enrolled in clinical trials, the patients in our study were younger, were more likely to be male, had a shorter duration of disease, and were less likely to be biologic naïve. Additional research is needed to better characterize baseline clinical features and to evaluate outcomes with secukinumab use as first-line biologic therapy for the treatment of AS in clinical practice versus clinical trials, but overall, these findings are among the first to provide rheumatologists with information on characteristics of and real-world dosing in patients with AS who initiate secukinumab in the US.

In this study, rheumatologists prescribed secukinumab to patients as maintenance therapy at the approved $150-\mathrm{mg}$ dose in more than one-half of patients with AS, with the remaining approximately $40 \%$ of patients receiving maintenance therapy at the higher $300-\mathrm{mg}$ dose. Similar proportions of patients in the Symphony database reported initiating secukinumab at doses of $150 \mathrm{mg}$ and $300 \mathrm{mg}(59.1 \%$ and $40.9 \%$, respectively) [15]. The safety and efficacy of secukinumab $300 \mathrm{mg}$ following an intravenous (IV) loading regimen was investigated in the phase III MEASURE 3 study, which found that the proportion of patients achieving an ASAS 20 response was significantly higher in both the IV-300 mg and IV-150 mg treatment groups than in the placebo group $(60.5 \%$ and $58.1 \%$ vs. $36.8 \%$; $p<0.05$ for both comparisons), with no differences in the overall safety profiles of the two dosing regimens [18]. A second study estimating the clinical difference between secukinumab $300 \mathrm{mg}$ and $150 \mathrm{mg}$ following dose escalation to $300 \mathrm{mg}$ (NCT03350815) is currently ongoing. It is possible that some physicians included in our study also treat patients with psoriatic arthritis and psoriasis and are comfortable with prescribing secukinumab at a dose of $300 \mathrm{mg}$. A dose-ranging study of secukinumab in patients with psoriasis found that patients weighing $<90 \mathrm{~kg}$ achieved better responses across all treatment groups compared with those patients weighing $\geq 90 \mathrm{~kg}$, suggesting a potential benefit of weight-based dosing. Although body mass index was not captured in our study, the increasing prevalence of obesity in the US population may contribute to increased use of 300-mg dosing in patients with AS seen in clinical practice [19]. Further research is needed to understand rheumatologist preferences for prescribing one dose over the other; however, the data suggest that in clinical practice for the treatment of AS, there may be flexibility in the dosing of secukinumab used.

The rheumatologists in our study had been in practice for approximately 13 years and most $(76.0 \%)$ used established criteria to assist in the diagnosis of AS. Although our retrospective chart review provided information on AS symptoms and co-morbidities, no information on specific measures of AS severity was available. Nevertheless, the proportion of patients with moderate AS in this study who initiated secukinumab is in line with recommendations to 
use biologics, including an IL-17 inhibitor, in patients who do not respond to nonbiologic therapies [7].

The most frequently cited reasons for secukinumab initiation among rheumatologists treating patients with AS were its efficacy/effectiveness, failure of other biologics, and its status as a newly available agent with a new mechanism of action. Although data on some AS symptoms, such as pain and stiffness, were available in this study, other key patientreported outcome measures were not available in medical charts, which otherwise might have provided additional information and insight into patient experiences to support reasons why secukinumab was prescribed and/or why prior treatments were discontinued [20-22]. Among patients with AS who were biologic naïve, efficacy/effectiveness remained the most common reason for secukinumab initiation, while failure of other prior biologics was the most common reason among biologic-experienced patients. Other reasons were fairly balanced between the two groups, including the desire to initiate a newly available treatment agent with a new mechanism of action. These reasons were primarily focused on the benefits related to efficacy, safety, and tolerability of treatment that have been demonstrated in randomized clinical trials $[16,18]$. Additionally, concerns about secukinumab-associated adverse events and insurance-related issues were less frequently cited than other reasons, suggesting that reported payer barriers were not particularly high or were deemed a lower priority. The decision to discontinue treatment could potentially be due to clinical versus insurance reasons. At the time of chart abstraction, patients had received secukinumab for a mean (SD) of 4.7 (4.6) months- this is comparable in duration to the 16-week placebo-controlled period of the MEASURE 1 and 2 pivotal clinical trials of secukinumab in patients with AS. Only three patients in our study had discontinued treatment at the time of chart abstraction, none of whom reported adverse events as a reason for discontinuation. By comparison, at week 16, 1.2\% of secukinumab-treated patients in MEASURE 1 and $4.8 \%$ of secukinumab-treated patients in MEASURE 2 discontinued treatment due to adverse events. It is possible that these rates could have been elevated because patients and investigators were blinded to study treatment in clinical trials. In addition, discontinuations due to adverse events may have been observed in our study with longer follow-up [16].

As with any retrospective observational study, our study has some limitations that should be considered when interpreting the results. The sample size was relatively small $(N=78)$, particularly for patients with milder rheumatologist-assessed disease severity, and this may reduce the generalizability of the findings to a larger US population of patients with AS; however, all regions of the US were well represented by participating rheumatologists. Because the study relied on self-report by the participating rheumatologists, these findings may be affected by reporting bias or recall bias in the physician screening portion of the study (e.g., bias in favor of secukinumab use, bias in favor of specific practice per guideline recommendations, or selection of "unknown/not sure" response options), as well as non-random missing data in the chart abstraction forms. In addition, rheumatologists contributed data from their own eligible patients into the study; while rheumatologists were to include all eligible patients, this may have been affected by recall bias from the rheumatologist and/or selection bias. Although patients' medical charts included information on AS diagnosis and three-quarters of rheumatologists used established criteria to aid in the diagnosis of AS, HLA-B27 was not collected by the chart abstraction forms, potentially limiting the generalizability of data to patients with confirmed AS. Furthermore, assessments of disease severity and treatment effectiveness in real-world settings may have been based on clinical expert opinion, using heterogeneous criteria, rather than validated and/or quantifiable measures of disease activity or severity. Lastly, although this study only included patients who initiated secukinumab, the choice of biologic and the decision of when to use secukinumab may differ based on the formulary of each payer/employer group. Despite these limitations, our study offers rheumatologists timely clinical data on patients with AS who initiated secukinumab. Future studies with proper follow-up will allow for additional real-world insights into the duration of secukinumab use, clinical outcomes, and patient-reported experiences while receiving treatment among patients with AS.

\section{Conclusion}

These findings provide insight into the characteristics of patients with AS in US clinical practice who are initiating secukinumab, the first and only non-TNFi approved for the treatment of AS in the US, within the first 2 years of approval and the factors leading to prescription of secukinumab by their treating rheumatologists. Approximately one-third of patients were biologic naïve and one-half of patients had received only one biologic prior to initiating secukinumab, which is surprising, given formulary restrictions typically imposed on newer biologic therapies. Lack/ loss of efficacy/effectiveness and disease progression/failure of other biologics were the most common rheumatologistreported reasons for discontinuing preceding treatments and initiating secukinumab in patients with AS. Because more than one-half of patients in our study received secukinumab at an initial loading dose of $300 \mathrm{mg}$, and $>40 \%$ of patients received secukinumab at a maintenance dose of $300 \mathrm{mg}$, future studies are needed to understand the dosing considerations weighed by rheumatologists who prescribe secukinumab for the treatment of AS. 
Acknowledgements Support for third-party writing assistance for this manuscript, furnished by Eric Deutsch, Ph.D., CMPP, of Health Interactions, Inc, was provided by Novartis Pharmaceuticals Corporation, East Hanover, NJ, USA.

\section{Compliance with Ethical Standards}

Funding This study was sponsored by Novartis Pharmaceuticals Corporation, East Hanover, NJ.

Conflict of interest R. Joshi has received consulting fees from Novartis. D. Latremouille-Viau, M. K. Meiselbach, and J. Xie are employees of Analysis Group, Inc. Y. Park is an employee of Novartis Pharmaceuticals Corporation. P. Sunkureddi has received consulting and speakers' bureau fees from Novartis, and is an investigator of a clinical trial sponsored by Novartis.

Research involving human participants An institutional review board exemption was obtained for the study prior to initiation of data collection.

Informed consent Informed consent was not required in this study because the data collected did not contain personal identifiers.

Open Access This article is distributed under the terms of the Creative Commons Attribution-NonCommercial 4.0 International License (http://creativecommons.org/licenses/by-nc/4.0/), which permits any noncommercial use, distribution, and reproduction in any medium, provided you give appropriate credit to the original author(s) and the source, provide a link to the Creative Commons license, and indicate if changes were made.

\section{References}

1. Taurog JD, Chhabra A, Colbert RA. Ankylosing spondylitis and axial spondyloarthritis. N Engl J Med. 2016;374:2563-74.

2. Braun J, Sieper J. Ankylosing spondylitis. Lancet. 2007;369:1379-90.

3. Reveille JD. Epidemiology of spondyloarthritis in North America. Am J Med Sci. 2011;341:284-6.

4. Reveille JD, Witter JP, Weisman MH. Prevalence of axial spondylarthritis in the United States: estimates from a cross-sectional survey. Arthritis Care Res (Hoboken). 2012;64:905-10.

5. Reveille JD, Weisman MH. The epidemiology of back pain, axial spondyloarthritis and HLA-B27 in the United States. Am J Med Sci. 2013;345:431-6.

6. Ward MM, Deodhar A, Akl EA, et al. American College of Rheumatology/Spondylitis Association of America/Spondyloarthritis Research and Treatment Network 2015 recommendations for the treatment of ankylosing spondylitis and nonradiographic axial spondyloarthritis. Arthritis Rheumatol. 2016;68:282-98.

7. van der Heijde D, Ramiro S, Landewe R, et al. 2016 update of the ASAS-EULAR management recommendations for axial spondyloarthritis. Ann Rheum Dis. 2017;76:978-91.
8. Sepriano A, Regel A, van der Heijde D, et al. Efficacy and safety of biological and targeted-synthetic DMARDs: a systematic literature review informing the 2016 update of the ASAS/EULAR recommendations for the management of axial spondyloarthritis. RMD Open. 2017;3:e000396 (2016-000396, eCollection 2017).

9. Deodhar A, Yu D. Switching tumor necrosis factor inhibitors in the treatment of axial spondyloarthritis. Semin Arthritis Rheum. 2017;47:343-50.

10. Ciurea A, Exer P, Weber U, et al. Does the reason for discontinuation of a first TNF inhibitor influence the effectiveness of a second TNF inhibitor in axial spondyloarthritis? Results from the Swiss Clinical Quality Management Cohort. Arthritis Res Ther. 2016;18:71016-0969-2.

11. Lie E, van der Heijde D, Uhlig T, et al. Effectiveness of switching between TNF inhibitors in ankylosing spondylitis: data from the NOR-DMARD register. Ann Rheum Dis. 2011;70:157-63.

12. ISPE. Guidelines for good pharmacoepidemiology practices (GPP). Pharmacoepidemiol Drug Saf. 2008;17:200-8.

13. von Elm E, Altman DG, Egger M, et al. The Strengthening the Reporting of Observational Studies in Epidemiology (STROBE) statement: guidelines for reporting observational studies. J Clin Epidemiol. 2008;61:344-9.

14. Magrey M, Bozyczko M, Wolin D, et al. Treatment experience and satisfaction in ankylosing spondylitis patients treated with secukinumab: results from a US web-based survey [abstract]. Ann Rheum Dis. 2018;77:1014 (SAT0300).

15. Oelke KR, Garg R, Li Y, et al. Real-world use of secukinumab among biologic-naïve and biologic-experienced patients with ankylosing spondylitis in the United States [abstract]. Arthritis Rheumatol. 2017;69:A1524.

16. Baeten D, Sieper J, Braun J, et al. Secukinumab, an interleukin-17A inhibitor, in ankylosing spondylitis. N Engl J Med. 2015;373:2534-48.

17. Sieper J, Deodhar A, Marzo-Ortega H, et al. Secukinumab efficacy in anti-TNF-naïve and anti-TNF-experienced subjects with active ankylosing spondylitis: results from the MEASURE 2 Study. Ann Rheum Dis. 2017;76:571-92.

18. Pavelka K, Kivitz A, Dokoupilova E, et al. Efficacy, safety, and tolerability of secukinumab in patients with active ankylosing spondylitis: a randomized, double-blind phase 3 study, MEASURE 3. Arthritis Res Ther. 2017;19:285.

19. Papp KA, Langley RG, Sigurgeirsson B, et al. Efficacy and safety of secukinumab in the treatment of moderate-to-severe plaque psoriasis: a randomized, double-blind, placebo-controlled phase II dose-ranging study. Br J Dermatol. 2013;168:412-21.

20. Deodhar A, Mittal M, Reilly P, et al. Ankylosing spondylitis diagnosis in US patients with back pain: identifying providers involved and factors associated with rheumatology referral delay. Clin Rheumatol. 2016;35:1769-76.

21. Danve A, Deodhar A. Screening and referral for axial spondyloarthritis-need of the hour. Clin Rheumatol. 2015;34:987-93.

22. López-Medina C, Garrido-Castro JL, Castro-Jiménez J, et al. Evaluation of quality of life in patients with axial spondyloarthritis and its association with disease activity, functionality, mobility, and structural damage. Clin Rheumatol. 2018;37:1581-8. 